\title{
A GAMIFIED, BLOCKCHAIN BASED, LEARNING RECORD STORE AS A PERSONAL LEARNING ENVIRONMENT COULD OFFER A SOLUTION TO THE HUMAN RIGHT OF EDUCATION
}

\author{
G. Turkawka \\ Zurich University of Arts, Zurich University of Teacher Education (SWITZERLAND) \\ gregory.turkawka@phzh.ch
}

Although universal digital identification systems are explored by many and personal learning record stores as part of LTI or XAPI collectors do exist, the creation of a global digital identification systems is currently contested by Google, Microsoft and Facebook as most widely used 3rd-party authentication systems. Also open access to educational resources is despite the digitalization a dream for many.

Aggregating formal and informal learning data and collecting them in a personal learning record store is a very sensitive issue and can't be trustworthy offered by companies or countries. This might be a reason there are currently no initiatives that succeed to implement a global system of digital identification nor a personal learning record store for everyone.

Creating a biometric digital identity verification system to grant access to a personal learning record store where informal and formal learning achievements get aggregated by XAPI-protocol, is building the ground for further use. An artificially intelligent tokenized real value-backed platform that analyzes personal achievements, badges and certificates and further learning opportunities according to culture and field of experience will offer additional educational resources, job opportunities or new fields of interest.

The combination of institutional or open educational resources and informal learning opportunities with selectable educational achievement maps and a tokenized value systems for further access to payed learning resources has not been realized yet. By creating a token system that is backed up with real funds as part of a personal learning record store, also the un-banked population can experience learning as value and get invested in further educational resources. Achievement maps and a non-biased evaluation and matching-system will give orientation and offer individual, personalized learning paths for further exploration as well as new possibilities to proof or certify achievements and competences.

By constantly adding new resources, learning results and achievements the data can be crunched and analyzed by artificial intelligent systems to offer clusters, trends and insights. The results in combination with an engaged community of learners can be used for further, also collaborative learning, the creation of new educational resources or for payed projects, job recruitment and job offerings. The semantic matching-system will help to find fits according to industry according to its cultural backgrounds. A blockchain as ledger will store all sensible data and build the trusted system for the users.

The concept is part of a Master thesis at Zurich University of Arts and Zurich University of Teacher Education. It should be shared and further developed in the sense of "Working Out Loud" together with interested individuals, organizations, developers, educators, NGOs and learners. The realization of a working prototype is planed early 2020.

Keywords: LMS, Learning Record Store, LRS, OER, SDG-4, AI, Blockchain, Digital ID, Certificates, xAPI, Batches, Matching-System, Achievement Maps, Gamification, PLE. 